\title{
Prevalência do ectrópio palpebral e características dos portadores na população da região Centro-Oeste do Estado de São Paulo
}

\author{
Eyelid ectropium prevalence and carriers characteristics in a population from \\ the Center-Western region of São Paulo State
}

\author{
Cristiane do Prado Silva ${ }^{1}$ \\ Silvana Artioli Schellini ${ }^{2}$ \\ Carlos RobertoPadovani ${ }^{3}$ \\ Silvia Narikawa ${ }^{4}$
}

\section{RESUMO}

Objetivo: Apresentar as características dos portadores e a prevalência do ectrópio em uma amostra populacional aleatória do Estado de São Paulo, Brasil. Material e Métodos: Os dados foram colhidos durante a realização do Projeto de Prevenção da Cegueira da Faculdade de Medicina de Botucatu - UNESP, com amostra domiciliar sistemática aleatória. Foram avaliados 10.432 indivíduos, residentes em 11 municípios da regional de saúde localizada na região Centro-Oeste do Estado de São Paulo. Os resultados obtidos foram submetidos a análise estatística para avaliação de variáveis descritivas e da prevalência do ectrópio palpebral. Resultados: Foram detectados 71 portadores de ectrópio palpebral, com prevalência estimada de $0,68 \%$. A prevalência não foi semelhante nos Municípios estudados. A maioria dos portadores de ectrópio apresentava idade acima dos 70 anos e era do sexo masculino. Vinte e oito por cento dos portadores de ectrópio apresentavam catarata concomitantemente. Conclusão: A prevalência do ectrópio na população CentroOeste do Estado de São Paulo é de 0,68\%. O ectrópio palpebral ocorre mais frequentemente em homens, geralmente idosos, expostos ao sol, mais na pálpebra inferior e com acuidade visual corrigida normal.

Descritores: Ectrópio/epidemiologia; Oftalmopatias/epidemiologia; Prevalência; Acuidade visual; Brasil/epidemiologia

\section{INTRODUÇÃO}

Ectrópio palpebral corresponde à eversão da pálpebra inferior, podendo ser de causa congênita ou adquirida, sendo as formas congênitas raras ${ }^{(1)}$.

Dentre as formas adquiridas, pode-se ter o tipo involucional (também conhecido como ectrópio senil, relacionado a alterações do envelhecimento), o cicatricial (decorrente de alterações cicatriciais da pele palpebral, muitas vezes induzidas pela radiação solar em portadores de pele clara), o mecânico (que ocorre por lesão que traciona mecanicamente a pálpebra para baixo), o paralítico (decorrente de paralisia inervacional da pálpebra) e o inflamatório (que ocorre por processos inflamatórios da margem palpebral). Dentre estas formas clínicas, a mais frequente é a involucional, que acomete a população de indivíduos idosos.

O envelhecimento resulta de inúmeros danos metabólicos e ambientais que acontecem ao longo de anos, causando vários distúrbios nos olhos da população idosa ${ }^{(2)}$, podendo o ectrópio estar presente, em associação com outras afecções. 
Entretanto, a idade não é fator isolado para o desenvolvimento do ectrópio, já que ele não ocorre em toda a população de idosos. Outras alterações são necessárias para produzir a eversão palpebral, alguns relacionados com fatores do próprio indivíduo e outros relacionados com o meio ambiente. Assim, outros importantes fatores para o desenvolvimento dessa afecção seriam: exposição à radiação solar, doenças palpebrais, cirurgias prévias, doenças neuromusculares e cerebrovasculares ${ }^{(3)}$.

O posicionamento incorreto das pálpebras no portador de ectrópio pode resultar em lagoftalmo, alterações corneanas pela oclusão incompleta das pálpebras, desordens da drenagem lacrimal, blefarite e déficit cosmético ${ }^{(3-4)}$.

O ectrópio é frequentemente observado nos consultórios e nos ambulatórios de especialidades oftalmológicas. Observação pessoal sugere ser este o tipo de alteração da posição palpebral mais frequente em nosso meio. Entretanto, não se conhece a distribuição desta afecção na população geral. Ademais, a situação de problemas na população geral dificilmente pode ser estimada, uma vez que estudos populacionais são poucos realizados, ficando-se mais com dados obtidos em hospitais, serviços especializados e estudos feitos em grupos populacionais específicos.

Na região Centro-Oeste do Estado de São Paulo foi realizado um estudo utilizando amostragem populacional, com o intuito de detectar problemas visuais na população. Os indivíduos que participaram do estudo foram escolhidos de forma aleatória e o exame dos mesmos permitiu detectar a prevalência de problemas oftalmológicos na população de estudo.

O presente trabalho tem como objetivo apresentar o perfil do portador e a prevalência de ectrópio nesta amostra populacional aleatória.

\section{MÉTODO}

Este estudo foi realizado baseando-se nos dados colhidos durante a realização do Projeto de Prevenção da Cegueira da Faculdade de Medicina de Botucatu - UNESP, desenvolvido utilizando um veículo do tipo unidade móvel oftalmológica, que abordou uma amostra sistemática domiciliar aleatória, nos anos de 2004 até 2006. Foram avaliados 10.432 indivíduos, residentes em 11 municípios da região Centro-Oeste do Estado de São Paulo, Brasil, a saber: Arandu, Areiópolis, Bofete, Botucatu, Conchas, Manduri, Pereiras, Piraju, Pratânea, São Manoel e Taguaí.

Todos os participantes foram submetidos a exame oftalmológico completo, feito por oftalmologistas ou residentes de oftalmologia, sendo os dados: idade, sexo, profissão, acuidade visual, exame externo, refratometria, biomicroscopia, fundoscopia, tonometria de aplanação, diagnóstico oftalmológico e tratamento, registrados em prontuário eletrônico.

Foram definidos como casos os indivíduos que apresentavam ectrópio palpebral ao exame externo.
Os resultados obtidos foram submetidos à análise estatística para avaliação de variáveis descritivas e estudo de prevalência do ectrópio palpebral na população geral.

\section{RESULTADOS}

Foram avaliados 10.432 indivíduos, tendo sido detectados 71 portadores de ectrópio palpebral, o que confere prevalência estimada de $0,68 \%$ de ectrópio na população estudada. A distribuição dos casos dentro dos municípios está apresentada na tabela 1, sendo possível observar que a possibilidade de se ter indivíduos com ectrópio na população foi diferente entre os municípios avaliados.

Quarenta e três $(60,56 \%)$ indivíduos que apresentaram ectrópio palpebral eram do sexo masculino e 28 (39,43\%), do sexo feminino, com diferença estatística entre os sexos (Gráfico 1).

Trinta e nove (55\%) portadores de ectrópio apresentavam idade acima dos 70 anos, seguindo-se por $22(31 \%)$ que estavam com idade entre 60 e 69 anos. Ou seja, $86 \%$ dos portadores de ectrópio tinham mais que 60 anos de idade (Gráfico 2).

O ectrópio era do tipo involucional $(25,35 \%)$, cicatricial $(11,27)$, ou misto $(11,27 \%)$. Considerou-se como ectrópio misto a alteração de posicionamento palpebral resultante da com-

\begin{tabular}{|lrr|}
\hline \multicolumn{3}{|c|}{$\begin{array}{c}\text { Tabela } \\
\text { 1. Prevalência de ectrópio palpebral na região Centro- } \\
\text { Oeste do Estado de São Paulo - UNESP, 2008 }\end{array}$} \\
Municípios & $\begin{array}{c}\text { Portadores } \\
\text { de ectrópio }\end{array}$ & $\begin{array}{c}\text { No total de indivíduos } \\
\text { atendidos }\end{array}$ \\
Arandu & $3(0,40 \%)$ & $746(100 \%)$ \\
Areiópolis & $4(0,52 \%)$ & $758(100 \%)$ \\
Bofete & $2(0,29 \%)$ & $692(100 \%)$ \\
Botucatu & $18(0,70 \%)$ & $2.554(100 \%)$ \\
Conchas & $15(1,48 \%)$ & $1.013(100 \%)$ \\
Manduri & $4(0,39 \%)$ & $1.020(100 \%)$ \\
Pereiras & $10(1,12 \%)$ & $895(100 \%)$ \\
Piraju & $4(0,50 \%)$ & $800(100 \%)$ \\
Pratânia & $6(0,86 \%)$ & $697(100 \%)$ \\
São Manoel & $0(0 \%)$ & $444(100 \%)$ \\
Taguaí & $5(0,61 \%)$ & $813(100 \%)$ \\
Total & $71(0,68 \%)$ & $10.432(100 \%)$ \\
\hline
\end{tabular}

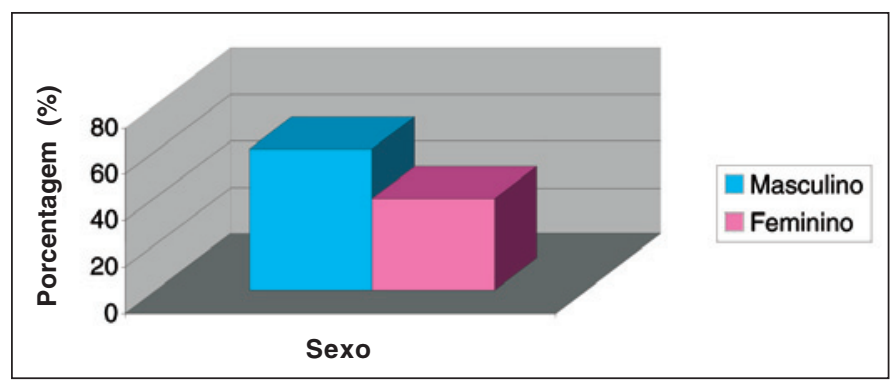

Gráfico 1 - Distribuição em porcentagem dos portadores de ectrópio palpebral residentes na região Centro-Oeste do Estado de São Paulo, de acordo com o sexo - UNESP, 2008 
binação de ectrópio involucional e cicatricial (Tabela 2). Não houve nenhum caso de ectrópio congênito simples ou associado com síndromes, como a blefarofimose.

Dos 71 casos estudados, foi possível saber ao certo da atividade profissional de apenas $37(52,11 \%)$. O restante informava a condição de aposentadoria, não categorizando a profissão exercida antes desta condição. Destes 37 portadores de ectrópio, $62 \%$ trabalhavam expostos ao sol.

Setenta por cento dos casos apresentava ectrópio bilateral, afetando a pálpebra inferior em todos eles. Entretanto, apesar de bilateral, o acometimento não foi simétrico na grande maioria dos indivíduos.

A acuidade visual corrigida segundo a tabela de Snellen foi maior que $0,7 \mathrm{em} 54,22 \%$ dos olhos avaliados. Deficiência visual esteve presente em $11,26 \%$ e cegueira em $8,45 \%$ dos olhos de portadores de ectrópio (Tabela 3 ).

Devido ao fato de se ter detectado ectrópio em idosos, a avaliação de alterações concomitantes mostrou que 98,59\%

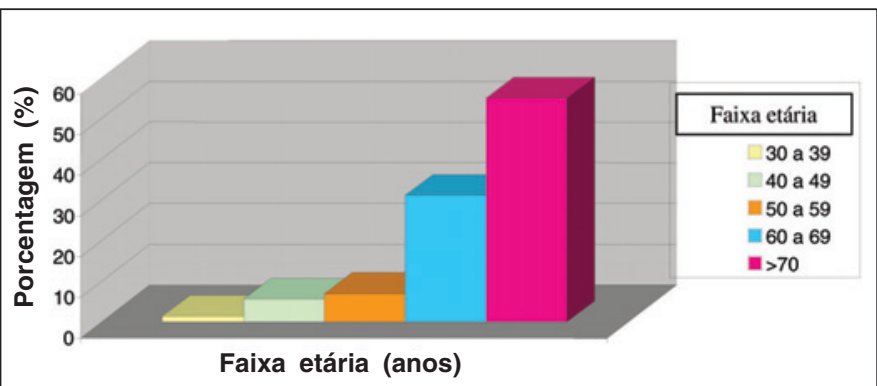

Gráfico 2 - Distribuição em porcentagem dos portadores de ectrópio palpebral da região Centro-Oeste do Estado de São Paulo, de acordo com a faixa etária (anos) - UNESP, 2008

Tabela 2. Classificação de ectrópio palpebral na população da região Centro-Oeste do Estado de São Paulo - UNESP, 2008

\begin{tabular}{lcc|} 
Diagnóstico ectrópio & Casos & Porcentagem (\%) \\
Ectrópio involucional & 18 & 25,35 \\
Ectrópio cicatricial & 8 & 11,27 \\
Ectrópio misto & 8 & 11,27 \\
Sem classificação & 37 & 52,11 \\
Total & 71 & 100,00 \\
\hline
\end{tabular}

Tabela 3. Distribuição a acuidade visual corrigida segundo número de olhos examinados, nos portadores de ectrópio palpebral da região Centro-Oeste do Estado de São Paulo - UNESP, 2008

\begin{tabular}{lrr}
$\begin{array}{l}\text { Acuidade visual com } \\
\text { correção }\end{array}$ & Casos & Porcentagem (\%) \\
\hline Não avaliada & 10 & 7,04 \\
$\geq 0,7$ & 77 & 54,22 \\
$0,3 \dashv 0,7$ & 27 & 19,01 \\
$0,3 \dashv 0,05$ & 16 & 11,26 \\
$\leq 0,05$ & 12 & 8,45 \\
Total & 142 & 100,00 \\
\hline
\end{tabular}

dos casos apresentavam concomitantemente presbiopia. Provavelmente devido a faixa etária afetada, a associação do ectrópio com a catarata esteve presente em $28,17 \%$ dos casos. $\mathrm{O}$ erro refracional mais observado nos portadores de ectrópio foi o astigmatismo hipermetrópico, presente em 50,70\% dos casos. Portanto, presbiopia, astigmatismo hipermetrópico e catarata foram os diagnósticos mais frequentes em concomitância com o ectrópio. Alguns casos apresentaram, ainda, pterígio $(22,54 \%)$, cílios malposicionados $(11,27 \%)$ ou degeneração macular relacionada à idade $(8,47 \%)$ (Tabela 4$)$.

\section{DISCUSSÃO}

Apesar de o ectrópio palpebral ser uma afecção frequentemente observada na prática oftalmológica, o presente estudo mostrou que a prevalência do ectrópio na população geral é relativamente baixa. A importância do estudo aqui apresentado está na avaliação de uma população escolhida de forma aleatória, com exame feito por oftalmologistas e com exame oftalmológico completo. Caso fosse realizada a pesquisa em uma população apenas de idosos ou em consultórios e serviços de referência, ou com exames incompletos, a prevalência provavelmente seria diferente, como se pode constatar pelo estudo da prevalência de ectrópio involucional em indivíduos acima de 80 anos, apontada como $69,5 \%$, enfatizando-se ser esta afecção a mais importante dentre as apresentadas pelos $\operatorname{idosos}^{(2)}$.

Algumas das cidades avaliadas apresentaram mais casos de ectrópio, o que pode estar associado ao tipo de atividade preponderante no local.

Os homens foram mais acometidos que as mulheres, com uma proporção de 1,5 homem:1 mulher. É consenso que os homens são mais propensos a apresentar afecções oculares e dos anexos, talvez por exercerem profissões mais expostas aos fatores de risco $^{(2)}$. Além disso, com relação especificamente ao ectrópio involucional, parece que os homens são mais afetados por apresentarem as dimensões do tarso maiores que as mulheres ${ }^{(5,7)}$.

\begin{tabular}{|c|c|c|}
\hline $\begin{array}{l}\text { Diagnósticos } \\
\text { concomitantes }\end{array}$ & $\begin{array}{l}\text { Número } \\
\text { de casos }\end{array}$ & $\begin{array}{c}\text { Porcentagem } \\
(\%)\end{array}$ \\
\hline Presbiopia & 70 & 98,59 \\
\hline Astigmatismo hipermetrópico & 36 & 50,70 \\
\hline Catarata & 20 & 28,17 \\
\hline Pterígio & 16 & 22,54 \\
\hline Malposicionamento dos cílios & 8 & 11,27 \\
\hline DMRI & 6 & 8,47 \\
\hline Total & $156^{*}$ & 100,00 \\
\hline \multicolumn{3}{|c|}{$\begin{array}{l}\text { DMRI= degeneração macular relacionada à idade } \\
{ }^{*}=0 \text { total é superior ao número de casos por ser possível mais de uma afecção } \\
\text { em um mesmo indivíduo }\end{array}$} \\
\hline
\end{tabular}


O dados observados mostraram que a grande maioria dos portadores de ectrópio são idosos, possuindo idade de 60 anos ou mais. A positiva relação do ectrópio com a idade (presente na maioria dos trabalhos que abordam esse assunto) provavelmente decorre da atrofia da placa tarsal, frouxidão tarso-ligamentar e presença de músculo orbicular forte, além de outras fenômenos involucionais que se instalam na senilidade ${ }^{(5-7)}$.

O ectrópio involucional foi o mais detectado na população de estudo, sendo este fato presente também em outros estu$\operatorname{dos}^{(3,7)}$. A ausência de ectrópio congênito nessa amostra aleatória reforça a raridade desta condição e realça ainda mais a importância do ectrópio involucional.

Além da idade, alguns outros fatores são considerados importantes para o desenvolvimento do ectrópio, tais como: o clima, as condições socioeconômicas e a profissãa ${ }^{(2)}$.

Neste ponto vale comentar que foi possível identificar a profissão de cerca de metade dos portadores de ectrópio, uma vez que a maioria referia a condição de aposentadoria, não especificando a atividade exercida antes disso. Importante notar que quase $70 \%$ dos casos em que se pode comprovar a atividade profissional, a exposição solar estava presente, reforçando a forte relação do ectrópio com a exposição $\operatorname{solar}^{(3)}$.

Devido a possibilidade de desenvolvimento da afecção segundo a atividade laborativa, explica-se a maior ocorrência do ectrópio em determinados municípios, com diferença estatística. Por se tratar, em geral, de pequenas cidades, a atividade profissional dos moradores das localidades estudadas muitas vezes é semelhante, tornando o risco para o desenvolvimento do ectrópio provavelmente ligado à atividade profissional daquela localidade.

O ectrópio é uma condição caracterizada pela eversão da margem palpebral, podendo ser bilateral e afetar ambas as pálpebras. Entretanto, a pálpebra inferior é mais frequentemente acometida, fato constatado em todos os portadores por nós estudados.

Em associação com o ectrópio, a catarata, o astigmatismo hipermetrópico e a presbiopia foram os diagnósticos mais frequentes. Estas afecções estão presentes nos indivíduos idosos, provavelmente o fator que levou à concomitância. Outras condições também associadas foram o pterígio (problema relacionado também com a exposição solar) e o malposicionamento dos cílios palpebrais, muitas vezes decorrente da inflamação crônica da margem ectropiada ${ }^{(4)}$.

\section{CONCLUSÃO}

O ectrópio palpebral esteve presente em $0,68 \%$ da população da região Centro-Oeste do Estado de São Paulo. Acomete preferencialmente os homens, em geral idosos (ectrópio involucional), sujeitos a exposição solar, mais frequente na pálpebra inferior e com acuidade visual normal .

\section{ABSTRACT}

Purpose: To present the characteristics of individuals and the prevalence of ectropium eyelid in a random sample of the state of São Paulo, Brazil. Methods: Data were collected during the Project on Prevention of Blindness, Faculty of Medicine of Botucatu - UNESP, a systematic random household sample was obtained. We evaluated 10,432 individuals, residents in 11 cities of the health regional located in the Central-Western region of the state of São Paulo. The results were submitted to statistical analysis to evaluate descriptive variables and the prevalence of ectropium eyelid. Results: Seventy-one ectropium eyelid patients were detected, with an estimated prevalence of $0.68 \%$. The prevalence was not similar in all the studied municipalities. Most people with ectropium eyelid presented age over 70 years and were male. Cataract was concurrently present in $28.0 \%$ of them. Conclusion: The prevalence of ectropium population in the Central-Western region of São Paulo state is $0.68 \%$. The ectropium eyelid affect mainly male, over 70 years relating sun light exposition and with normal visual acuity.

Keywords: Ectropion/epidemiology; Eye diseases/epidemiology; Prevalence; Visual acuity; Brazil/epidemiology

\section{REFERÊNCIAS}

1. Cruz AAV, Chahud F, Guimarães FC. Patologias dos anexos oculares. Medicina-Ribeirão Preto. 1997;30:36-51

2. Romani FA. Prevalência de transtornos oculares na população de idosos residentes na cidade de Veranópolis - RS, Brasil. Arq Bras Oftalmol. 2005;68(5): 649-55

3. Mitchell P, Hinchcliffe P, Wang JJ, Rochtchina E, Foran S. Prevalence and associations with ectropion in an older population: the Blue Mountains Eye Study. Clin Experiment Ophthalmol. 2001;29(3):108-10

4. Schellini SA, Zimmermann GPM, Hoyama E, Padovani CR, Padovani CRP. Alterações da margem palpebral associadas ao ectrópio. Arq Bras Oftalmol. 2005;68(5):619-22

5. Bashour M, Harvey, J. Causes of involutional ectropion and entropion - agerelated tarsal changes are the key. Ophthal Plas Reconst Surg. 2000;16(2): 131-41.

6. Sisler HA, Labay GR, Finlay JR. Senile ectropion and entropion: a comparative histopathological study. Ann Ophthalmol. 1976;8(3):319-22.

7. Veloso CER, Schellini SA, Padovani CR, Padovani CRP. Ectrópio palpebral: características e relação com alterações óculo-palpebrais. Rev Bras Oftalmol. 2006;65(3):147-51. 